\title{
Use of sliding-scale insulin in medical patients at Hamad General Hospital, Qatar - an audit-based study
}

\author{
Authors: Raza Akbar, ${ }^{\mathrm{A}}$ Jessiya Parambil, ${ }^{\mathrm{A}}$ Saleema Purayil, ${ }^{\mathrm{A}}$ Mohammad Abutineh ${ }^{\mathrm{A}}$ and Abdul Qader ${ }^{\mathrm{A}}$
}

\section{Introduction}

Poor control of diabetes mellitus (DM) in hospitalised patients is associated with worse clinical outcomes. Despite the lack of evidence of effectiveness of sliding-scale insulin (SSI), it remains a common practice in hospital settings. ${ }^{1,2}$

The aim of this study was to evaluate current practice of SSI use in medical inpatients at Hamad General Hospital.

\section{Materials and methods}

Electronic medical records of 30 patients with diabetes admitted under medical care (June 2017) were examined retrospectively.

\section{Results and discussion}

> The majority (63.3\%) of patients were female, and elderly $(>60 ; 53.3 \%)$.

$>$ The majority $(70 \%)$ of patients had type-2 DM. The type of DM was not documented in the rest.

> SSI was initiated on first day of hospital admission in almost all patients.

> SSI was continued beyond 48 hours in almost $80 \%$ of patients.

> The indication for initiation of SSI was not documented in almost all the patients.

$>$ Regular insulin was used in $23.3 \%$ of patients and insulin aspart in the rest.

> Basal insulin was prescribed in only $53.3 \%$ of the patients. Of these, $68.75 \%$ were already on home basal insulin, either with oral diabetic agents or with bolus insulin.

$>$ Almost a quarter (23.3\%) of patients had blood glucose readings of $<5 \mathrm{mmol} / \mathrm{L}$. Of these, $28.6 \%$ had symptomatic hypoglycaemia.

> Almost a third (30\%) of the patients had high blood glucose readings ( $>20 \mathrm{mmol} / \mathrm{L})$.

$>58.6 \%$ of patients were discharged on their previous home treatment.

> 24 -hr insulin requirement was calculated only in $10 \%$ of patients.

\section{Conclusions}

> There is variable glycaemic control with the use of SSI.

> Hypoglycaemia and hyperglycaemia are common problems with SSI.

> The accurate documentation of indication for SSI, and equivalent dose conversion, is still lacking.

$>$ SSI is used well beyond the maximum suggested duration of 48 hrs.

$>$ We aim to implement necessary changes and plan to re-audit to assess these changes.

\section{Conflicts of interest}

None declared.

\section{References}

1 Lee YY, Lin YM, Leu WJ et al. Sliding-scale insulin used for blood glucose control: a meta-analysis of randomized controlled trials. Metabolism 2015;64:118-392.

2 Dickerson LM, Ye X, Sack J, Hueston WJ. Glycemic control in medical inpatients with type 2 diabetes mellitus receiving sliding scale insulin regimens versus routine diabetes medications: a multicenter randomized controlled trial. Ann Fam Med 2003;1:29-35. 